\title{
Estimation of a general parametric location in censored regression
}

\author{
Cédric Heuchenne * \\ QuantOM, ${ }^{\dagger}$ \\ HEC-Management School of University of Liège \\ and Institute of Statistics \\ Université catholique de Louvain
}

\author{
Ingrid VAN KEILEGOM $\ddagger$ \\ Institute of Statistics
}

Université catholique de Louvain

June 2, 2011

\begin{abstract}
Consider the random vector $(X, Y)$, where $Y$ represents a response variable and $X$ an explanatory variable. The response $Y$ is subject to random right censoring, whereas $X$ is completely observed. Let $m(x)$ be a conditional location function of $Y$ given $X=x$. In this paper we assume that $m(\cdot)$ belongs to some parametric class $\mathcal{M}=\left\{m_{\theta}: \theta \in \Theta\right\}$ and we propose a new method for estimating the true unknown value $\theta_{0}$. The method is based on nonparametric imputation for the censored observations. The consistency and asymptotic normality of the proposed estimator are established.
\end{abstract}

Key Words: Least-squares estimator; L-functionals; Nadaraya-Watson estimator; Nonlinear regression; Nonparametric regression; Right censoring; Survival analysis.

*Financial support from IAP research network P6/03 of the Belgian Government (Belgian Science Policy) is gratefully acknowledged.

${ }^{\dagger}$ Centre for Quantitative Methods and Operations Management

${ }^{\ddagger}$ Financial support from IAP research network P6/03 of the Belgian Government (Belgian Science Policy), and from the European Research Council under the European Community's Seventh Framework Programme (FP7/2007-2013) / ERC Grant agreement No. 203650 is gratefully acknowledged. 


\section{Introduction}

Consider the random vector $(X, Y)$, where $Y$ represents a (possible transformation of a) response variable and $X$ an explanatory variable. This paper is concerned with the estimation of a location functional of $Y$ given $X$, when $Y$ is subject to random right censoring and $X$ is completely observed. We suppose that this location functional belongs to some parametric family.

This problem has been widely studied in the literature when the location functional is the conditional mean (see e.g. Stute (1993), Fygenson and Zhou (1994), Van Keilegom and Akritas (2000) among many others) or the conditional median (see e.g. Buchinsky and Hahn (1998), Portnoy (2003), Yin et al (2008), Wang and Wang (2009) and the references therein). Here we focus attention on $L$-functionals, given by

$$
m(x)=\int_{0}^{1} F^{-1}(s \mid x) J(s) d s,
$$

where $F(y \mid x)=P(Y \leq y \mid X=x)$ is the conditional distribution of $Y$ given $X=x$, $F^{-1}(s \mid x)=\inf \{y: F(y \mid x) \geq s\}$ is the conditional quantile of order $s$, and $J(s)$ is a weight function satisfying $J(s) \geq 0$ for all $0 \leq s \leq 1$ and $\int_{0}^{1} J(s) d s=1$. This type of location functionals includes as special cases the conditional mean, trimmed mean, or any other kind of weighted mean. The conditional median can be regarded as a limiting special case, obtained when $J(s)$ puts all its mass on $s=1 / 2$.

Another interesting special case is obtained for $J(s)=I(1-\delta<s \leq 1) / \delta$ for some $0<\delta<1$. Suppose the upper bound of the support of $Y$ given $X=x$ is finite (say equal to $\tau_{x}$ ) and one is interested in the estimation of the support curve $x \rightarrow \tau_{x}$. The above choice of $J$ yields a robust estimator of this curve for small values of $\delta$, and is an interesting alternative to the so-called $m$-frontiers or $\alpha$-frontiers, which are based on order statistics of order $m$ or quantiles of order $0<\alpha<1$ (see e.g. Cazals et al (2002) and Aragon et al (2005)).

We suppose in this paper that $Y$ is subject to random right censoring, i.e. instead of observing $Y$ we only observe $(Z, \Delta)$, where $Z=\min (Y, C)$ is the observed survival time, $\Delta=I(Y \leq C)$ is the censoring indicator, and the random variable $C$ represents the censoring time, which is independent of $Y$ conditionally on $X$. Let $\left(X_{i}, Z_{i}, \Delta_{i}\right)(i=$ $1, \ldots, n)$ be $n$ independent copies of $(X, Z, \Delta)$.

In the context of regression with right censored responses it is well known that the nonparametric kernel estimator of the conditional distribution $F(\cdot \mid x)$ is inconsistent in the right tail. The modeling of the above location functional $m(x)$ is therefore especially 
attractive in this framework, since particular choices of $J$-functions enable us to get rid of these inconsistent parts (see Section 2 for more detatils).

The goal of this paper is to propose a new estimation method for $m(x)$, when it is believed that $m(\cdot)$ belongs to the class

$$
\mathcal{M}=\left\{m_{\theta}: \theta \in \Theta\right\}
$$

consisting of location functionals determined by a finite-dimensional parameter vector $\theta \in \Theta$, where $\Theta$ is a compact subset of $\mathbb{R}^{d}$. The class $\mathcal{M}$ can be taken equal to the class of polynomial functions of order $d-1$, but any other parametric class of "smooth" functions (in $\theta$ ) can be chosen as well.

The paper is organized as follows. In the next section we introduce some notation and explain in detail the proposed estimation procedure for $\theta_{0}$, the true unknown value of $\theta$. Section 3 gives the main asymptotic properties of the proposed estimator. In Section 4 we summarize the results of the paper and give ideas for future research, whereas the Appendix contains the proofs of the main asymptotic results.

\section{Description of the method}

The estimator $\theta_{n}$ is defined as follows. First, we note that $\theta_{0}$ can be written as

$$
\begin{aligned}
\theta_{0}=\operatorname{argmin}_{\theta} E\left[\left(\int_{0}^{1} F^{-1}(s \mid X) J(s) d s-m_{\theta}(X)\right)^{2}\right] \\
=\operatorname{argmin}_{\theta} E\left[\left(Y-m_{\theta}(X)\right)^{2} J(F(Y \mid X))\right] \\
=\operatorname{argmin}_{\theta} E\left[\left(Z-m_{\theta}(X)\right)^{2} J(F(Z \mid X)) \Delta\right. \\
\left.+\frac{\int_{Z}^{\infty}\left(y-m_{\theta}(X)\right)^{2} J(F(y \mid X)) d F(y \mid X)}{1-F(Z \mid X)}(1-\Delta)\right] .
\end{aligned}
$$

The idea is now to estimate $\theta_{0}$ by a minimizer $\theta_{n}$ of an empirical version of the above quantity, namely

$$
\begin{aligned}
\theta_{n}=\operatorname{argmin}_{\theta \in \Theta} n^{-1} \sum_{i=1}^{n}\left[\left(Z_{i}-m_{\theta}\left(X_{i}\right)\right)^{2} J\left(\hat{F}\left(Z_{i} \mid X_{i}\right)\right) \Delta_{i}\right. \\
\left.+\frac{\int_{Z_{i}}^{\infty}\left(y-m_{\theta}\left(X_{i}\right)\right)^{2} J\left(\hat{F}\left(y \mid X_{i}\right)\right) d \hat{F}\left(y \mid X_{i}\right)}{1-\hat{F}\left(Z_{i} \mid X_{i}\right)}\left(1-\Delta_{i}\right)\right] .
\end{aligned}
$$


Here, $\hat{F}(y \mid x)$ is the nonparametric kernel estimator of the conditional distribution $F(y \mid x)$ proposed by Beran (1981) :

$$
\hat{F}(y \mid x)=1-\prod_{Z_{i} \leq y, \Delta_{i}=1}\left\{1-\frac{W_{i}\left(x, a_{n}\right)}{\sum_{k=1}^{n} I\left(Z_{k} \geq Z_{i}\right) W_{k}\left(x, a_{n}\right)}\right\},
$$

(when no ties are present), where

$$
W_{i}\left(x, a_{n}\right)=\frac{K\left(\frac{x-X_{i}}{a_{n}}\right)}{\sum_{k=1}^{n} K\left(\frac{x-X_{k}}{a_{n}}\right)},
$$

$K$ is a kernel function and $\left\{a_{n}\right\}$ is a bandwidth sequence.

Hence, the estimation procedure for $\theta_{0}$ can be summarized as follows :

1. First, for fixed $\theta$, estimate the weighted squared error $\left(Z_{i}-m_{\theta}\left(X_{i}\right)\right)^{2} J\left(F\left(Z_{i} \mid X_{i}\right)\right)$ of an uncensored observation $\left(X_{i}, Y_{i}, \Delta_{i}=1\right)$ by $\left(Y_{i}-m_{\theta}\left(X_{i}\right)\right)^{2} J\left(\hat{F}\left(Y_{i} \mid X_{i}\right)\right)$, where $\hat{F}$ is defined in (2.2), and of a censored observation $\left(X_{i}, C_{i}, \Delta_{i}=0\right)$ by a nonparametric estimator of $E\left[\left(Y-m_{\theta}(X)\right)^{2} J(\hat{F}(Y \mid X)) \mid X=X_{i}, Y>C_{i}\right]$.

2. Then, estimate $\theta_{0}$ by minimizing the average of the weighted squared errors obtained under the previous step.

Although the above idea of estimating $\theta_{0}$ has never been considered in the present context of nonlinear parametric estimation of a general location functional, similar versions of this idea have been applied in other contexts. See e.g. Akritas (1996), who, in the context of polynomial regression, first replaced all observations $Z_{i}$ (censored and uncensored ones) by a nonparametric estimator $\widehat{m}\left(X_{i}\right)$ of $m\left(X_{i}\right)$, and then applied a classical least squares procedure on the so-obtained 'synthetic' data $\left(X_{i}, \widehat{m}\left(X_{i}\right)\right)$. His method has the disadvantage that it is quite sensitive to the choice of the bandwidth, as the bandwidth is playing an important role for both the censored and the uncensored data. Another related methodology is given in Heuchenne and Van Keilegom (2007), who consider the estimation of the conditional mean of $Y$ given $X$ when the relation between $Y$ and $X$ is given by a nonparametric location-scale model. They also replace the censored observations by some kind of synthetic data estimated under the assumed location-scale model. Also see Pardo-Fernández et al (2007) for a goodness-of-fit test in parametric censored regression.

For the presentation of the asymptotic results in the next section, we need to introduce the following notation. Let $H(y \mid x)=P(Z \leq y \mid X=x), H_{\delta}(y \mid x)=P(Z \leq y, \Delta=\delta \mid X=$ 
$x)(\delta=0,1), F_{\varepsilon}(y \mid x)=P(\varepsilon \leq y \mid X=x)$ and $F_{X}(x)=P(X \leq x)$. The probability density functions of the above distribution functions will be denoted by lower case letters. Also, let

$\epsilon_{\theta}(x, z, \delta, F)=\left\{\delta\left(z-m_{\theta}(x)\right)^{2} J(F(z \mid x))+(1-\delta) \frac{\int_{z}^{+\infty}\left(y-m_{\theta}(x)\right)^{2} J(F(y \mid x)) d F(y \mid x)}{1-F(z \mid x)}\right\}$

(where $E\left[\epsilon_{\theta}(X, Z, \Delta, F)\right]=E\left[\left(Y-m_{\theta}(X)\right)^{2} J(F(Y \mid X))\right]$ ), which implies that the estimator $\theta_{n}$ can be written as

$$
\theta_{n}=\operatorname{argmin}_{\theta \in \Theta} \sum_{i=1}^{n} \epsilon_{\theta}\left(X_{i}, Z_{i}, \Delta_{i}, \hat{F}\right)
$$

where $\hat{F}$ is the Beran estimator defined in (2.2).

\section{Asymptotic results}

We start by showing the convergence in probability of $\theta_{n}$ and of the least squares criterion function. This will allow us to develop an asymptotic representation for $\theta_{n j}-\theta_{0 j}(j=$ $1, \ldots, d)$, which in turn will give rise to the asymptotic normality of these estimators. The assumptions used in the results below, as well as the proof of the two first results, are given in the Appendix.

Theorem 3.1 Assume (A1), (A2) (i), (A4) (i), (iv), (v) and (A7). Moreover, assume that $J$ is continuously differentiable, $\int_{0}^{1} J(s) d s=1, J(s) \geq 0$ for all $0 \leq s \leq 1, F_{X}$ is two times continuously differentiable, $\inf _{x \in R_{X}} f_{X}(x)>0, \Theta$ is compact, $\theta_{0}$ is an interior point of $\Theta$, and $m_{\theta}(x)$ is continuous in $(x, \theta)$ for all $x$ and $\theta$. Let

$$
S_{n}(\theta)=\frac{1}{n} \sum_{i=1}^{n} \epsilon_{\theta}\left(X_{i}, Z_{i}, \Delta_{i}, \hat{F}\right)
$$

Then

$$
\theta_{n}-\theta_{0}=o_{P}(1)
$$

and

$$
S_{n}\left(\theta_{n}\right)=E\left[\epsilon_{\theta_{0}}(X, Z, \Delta, F)\right]+o_{P}(1)
$$

The next result decomposes the difference $\theta_{n}-\theta_{0}$ into a sum of i.i.d. terms and a negligible term of lower order. This decomposition will be crucial for obtaining the asymptotic normality of $\theta_{n}$. 
Theorem 3.2 Assume (A1)-(A7). Then,

$$
\theta_{n}-\theta_{0}=\Omega^{-1} n^{-1} \sum_{i=1}^{n} \rho\left(X_{i}, Z_{i}, \Delta_{i}\right)+\left(\begin{array}{c}
o_{P}\left(n^{-1 / 2}\right) \\
\vdots \\
o_{P}\left(n^{-1 / 2}\right)
\end{array}\right)
$$

where $\Omega=\left(\Omega_{j k}\right)(j, k=1, \ldots, d)$,

$$
\Omega_{j k}=E\left[\frac{\partial m_{\theta_{0}}(X)}{\partial \theta_{j}} \frac{\partial m_{\theta_{0}}(X)}{\partial \theta_{k}}\right],
$$

$\rho=\left(\rho_{1}, \ldots, \rho_{d}\right)^{T}$, and for any $j=1, \ldots, d$ and $i=1, \ldots, n$,

$$
\begin{aligned}
\rho_{j}\left(X_{i}, Z_{i}, \Delta_{i}\right)= & \frac{\partial m_{\theta_{0}}\left(X_{i}\right)}{\partial \theta_{j}}\left\{\Delta_{i}\left(Z_{i}-m_{\theta_{0}}\left(X_{i}\right)\right) J\left(F\left(Z_{i} \mid X_{i}\right)\right)\right. \\
& \left.+\left(1-\Delta_{i}\right) \frac{\int_{Z_{i}}^{+\infty}\left(y-m_{\theta_{0}}\left(X_{i}\right)\right) J\left(F\left(y \mid X_{i}\right)\right) d F\left(y \mid X_{i}\right)}{1-F\left(Z_{i} \mid X_{i}\right)}\right\} \\
& +f_{X}\left(X_{i}\right) \sum_{\delta=0,1} \int \chi_{j}\left(\left(X_{i}, z, \delta\right),\left(Z_{i}, \Delta_{i}\right)\right) d H_{\delta}\left(z \mid X_{i}\right),
\end{aligned}
$$

where the function $\chi_{j}$ is defined in the Appendix.

We are now ready to state the asymptotic normality of $\theta_{n}$.

Theorem 3.3 Under the assumptions of Theorem 3.2, $n^{1 / 2}\left(\theta_{n}-\theta_{0}\right) \stackrel{d}{\rightarrow} N(0, \Sigma)$, where

$$
\Sigma=\Omega^{-1} E\left[\rho(X, Z, \Delta) \rho^{T}(X, Z, \Delta)\right] \Omega^{-1}
$$

The proof of this result follows readily from Theorem 3.2.

\section{Summary and future research}

In this paper we have proposed a new method to estimate the coefficients of a parametric conditional location function, when the response is subject to random right censoring. The proposed estimator is a least squares type estimator, for which the censored observations are replaced by nonparametrically imputed values. The consistency and asymptotic normality of the estimator are established.

In the future, it would be interesting to compare the proposed method with other estimators that have been proposed in the literature, for instance, when the conditional location is the conditional mean. The least squares estimators obtained in this paper can 
be introduced in a test statistic to test the validity of the assumed parametric model, and it would be interesting to work out the asymptotic theory for that test statistic. Finally, extensions of the current work to semiparametric models (like the partial linear or single index model) can also be worked out based on the results in this paper.

\section{Appendix}

We first introduce the following functions, which are needed in the statement of the asymptotic results given in Section 3 :

$$
\begin{aligned}
& \xi(z, \delta, y \mid x)=(1-F(y \mid x))\left\{-\int_{-\infty}^{y \wedge z} \frac{d H_{1}(s \mid x)}{(1-H(s \mid x))^{2}}+\frac{I(z \leq y, \delta=1)}{1-H(z \mid x)}\right\}, \\
& \chi_{j}\left(v^{1}, z^{2}, \delta^{2}\right)= \frac{\partial m_{\theta_{0}}\left(x^{1}\right)}{\partial \theta_{j}}\left\{\left[\delta^{1}\left(z^{1}-m_{\theta_{0}}\left(x^{1}\right)\right) J^{\prime}\left(F\left(z^{1} \mid x^{1}\right)\right)\right.\right. \\
&+\left.\left(1-\delta^{1}\right) \frac{\int_{z^{1}}^{+\infty}\left(y-m_{\theta_{0}}\left(x^{1}\right)\right) J\left(F\left(y \mid x^{1}\right)\right) d F\left(y \mid x^{1}\right)}{\left(1-F\left(z^{1} \mid x^{1}\right)\right)^{2}}\right] \xi\left(z^{2}, \delta^{2}, z^{1} \mid x^{1}\right) \\
&+\left(1-\delta^{1}\right)\left[\frac{\int_{z^{1}}^{+\infty}\left(y-m_{\theta_{0}}\left(x^{1}\right)\right) J\left(F\left(y \mid x^{1}\right)\right) d \xi\left(z^{2}, \delta^{2}, y \mid x^{1}\right)}{1-F\left(z^{1} \mid x^{1}\right)}\right. {\left.\left[\frac{\int_{z^{1}}^{+\infty}\left(y-m_{\theta_{0}}\left(x^{1}\right)\right) J^{\prime}\left(F\left(y \mid x^{1}\right)\right) \xi\left(z^{2}, \delta^{2}, y \mid x^{1}\right) d F\left(y \mid x^{1}\right)}{1-F\left(z^{1} \mid x^{1}\right)}\right]\right\}, }
\end{aligned}
$$

$j=1, \ldots, d$, where $v^{1}=\left(x^{1}, z^{1}, \delta^{1}\right)$.

Let $T_{x}$ be any value less than the upper bound of the support of $H(\cdot \mid x)$ such that $\inf _{x \in R_{X}}\left(1-H\left(T_{x} \mid x\right)\right)>0$. For a (sub)distribution function $L(y \mid x)$ we will use the notations $l(y \mid x)=L^{\prime}(y \mid x)=(\partial / \partial y) L(y \mid x), \dot{L}(y \mid x)=(\partial / \partial x) L(y \mid x)$ and similar notations will be used for higher order derivatives.

The assumptions needed for the results of Section 3 are listed below.

$(A 1)(i) n a_{n}^{3}(\log n)^{-3} \rightarrow \infty$ and $n a_{n}^{4} \rightarrow 0$.

(ii) The support $R_{X}$ of $X$ is a compact interval.

(iii) $K$ is a density with compact support, $\int u K(u) d u=0$ and $K$ is twice continuously differentiable.

(iv) $\Omega$ is non-singular. 
$(A 2)(i)$ There exist $0 \leq s_{0} \leq s_{1} \leq 1$ such that $s_{1} \leq \inf _{x} F\left(T_{x} \mid x\right), s_{0} \leq \inf \{s \in$ $[0,1] ; J(s) \neq 0\}, s_{1} \geq \sup \{s \in[0,1] ; J(s) \neq 0\}$ and $\inf _{x \in R_{X}} \inf _{s_{0} \leq s \leq s_{1}} f\left(F^{-1}(s \mid x) \mid x\right)>0$.

(ii) $J$ is three times continuously differentiable, $\int_{0}^{1} J(s) d s=1$ and $J(s) \geq 0$ for all $0 \leq s \leq 1$.

(A3) $F_{X}$ is three times continuously differentiable and $\inf _{x \in R_{X}} f_{X}(x)>0$.

$(A 4)(i) L(y \mid x)$ is continuous,

(ii) $L^{\prime}(y \mid x)=l(y \mid x)$ exists, is continuous in $(x, y)$ and $\sup _{x, y}\left|y L^{\prime}(y \mid x)\right|<\infty$,

(iii) $L^{\prime \prime}(y \mid x)$ exists, is continuous in $(x, y)$ and $\sup _{x, y}\left|y^{2} L^{\prime \prime}(y \mid x)\right|<\infty$,

(iv) $\dot{L}(y \mid x)$ exists, is continuous in $(x, y)$ and $\sup _{x, y}|y \dot{L}(y \mid x)|<\infty$,

(v) $\ddot{L}(y \mid x)$ exists, is continuous in $(x, y)$ and $\sup _{x, y}\left|y^{2} \ddot{L}(y \mid x)\right|<\infty$,

(vi) $\ddot{L}^{\prime}(y \mid x)$ exists, is continuous in $(x, y)$ and $\sup _{x, y}\left|y \ddot{L}^{\prime}(y \mid x)\right|<\infty$,

for $L(y \mid x)=H(y \mid x)$ and $H_{1}(y \mid x)$.

$(A 5)$ For the density $f_{X \mid Z, \Delta}(x \mid z, \delta)$ of $X$ given $(Z, \Delta), \sup _{x, z}\left|f_{X \mid Z, \Delta}(x \mid z, \delta)\right|<\infty$, $\sup _{x, z}\left|\dot{f}_{X \mid Z, \Delta}(x \mid z, \delta)\right|<\infty, \sup _{x, z}\left|\ddot{f}_{X \mid Z, \Delta}(x \mid z, \delta)\right|<\infty(\delta=0,1)$.

(A6) $\Theta$ is compact and $\theta_{0}$ is an interior point of $\Theta$. All partial derivatives of $m_{\theta}(x)$ with respect to the components of $\theta$ up to order three exist and are continuous in $(x, \theta)$ for all $x$ and $\theta$.

(A7) The function $E\left[\left(Y-m_{\theta}(X)\right)^{2} J(F(Y \mid X))\right]$ has a unique minimum in $\theta=\theta_{0}$.

Proof of Theorem 3.1. We prove the consistency of $\theta_{n}$ by verifying the conditions of Theorem 5.7 in van der Vaart (1998, p. 45). From the definition of $\theta_{n}$ and condition $(A 7)$, it follows that it suffices to show that

$$
\sup _{\theta}\left|S_{n}(\theta)-S_{0}(\theta)\right| \rightarrow_{P} 0
$$

where $S_{0}(\theta)=E\left[\left(Y-m_{\theta}(X)\right)^{2} J(F(Y \mid X))\right]=E\left[\varepsilon^{2} J\left(F_{\varepsilon}(\varepsilon \mid X)\right)\right]$. The second statement of Theorem 3.1 then follows immediately from (A.1) together with the consistency of $\theta_{n}$. (A.1) is obtained by using (A2), the uniform consistency of the Beran (1981) estimator (given in Proposition A.3 of Van Keilegom and Akritas (1999)) and Theorem 2 of Jennrich (1969). 
Proof of Theorem 3.2. For some $\theta_{1 n}$ between $\theta_{n}$ and $\theta_{0}$,

$$
\theta_{n}-\theta_{0}=-\left\{\frac{\partial^{2} S_{n}\left(\theta_{1 n}\right)}{\partial \theta \partial \theta^{T}}\right\}^{-1} \frac{\partial S_{n}\left(\theta_{0}\right)}{\partial \theta}=-R_{1 n}^{-1} R_{2 n} .
$$

First, we treat $R_{2 n}$.

$$
\begin{aligned}
R_{2 n k}=- & \frac{2}{n} \sum_{i=1}^{n} \frac{\partial m_{\theta_{0}}\left(X_{i}\right)}{\partial \theta_{k}}\left\{\Delta_{i}\left(Z_{i}-m_{\theta_{0}}\left(X_{i}\right)\right) J^{\prime}\left(F\left(Z_{i} \mid X_{i}\right)\right)\left(\hat{F}\left(Z_{i} \mid X_{i}\right)-F\left(Z_{i} \mid X_{i}\right)\right)\right. \\
& +\left(1-\Delta_{i}\right)\left[\frac{\int_{Z_{i}}^{+\infty}\left(y-m_{\theta_{0}}\left(X_{i}\right)\right) J^{\prime}\left(F\left(y \mid X_{i}\right)\right)\left(\hat{F}\left(y \mid X_{i}\right)-F\left(y \mid X_{i}\right)\right) d F\left(y \mid X_{i}\right)}{1-F\left(Z_{i} \mid X_{i}\right)}\right. \\
& +\frac{\int_{Z_{i}}^{+\infty}\left(y-m_{\theta_{0}}\left(X_{i}\right)\right) J\left(F\left(y \mid X_{i}\right)\right) d F\left(y \mid X_{i}\right)}{\left(1-F\left(Z_{i} \mid X_{i}\right)\right)^{2}}\left(\hat{F}\left(Z_{i} \mid X_{i}\right)-F\left(Z_{i} \mid X_{i}\right)\right) \\
& \left.\left.+\frac{\int_{Z_{i}}^{+\infty}\left(y-m_{\theta_{0}}\left(X_{i}\right)\right) J\left(F\left(y \mid X_{i}\right)\right) d\left(\hat{F}\left(y \mid X_{i}\right)-F\left(y \mid X_{i}\right)\right)}{1-F\left(Z_{i} \mid X_{i}\right)}\right]\right\} \\
& -\frac{2}{n} \sum_{i=1}^{n} \frac{\partial m_{\theta_{0}}\left(X_{i}\right)}{\partial \theta_{k}}\left\{\Delta_{i}\left(Z_{i}-m_{\theta_{0}}\left(X_{i}\right)\right) J\left(F\left(Z_{i} \mid X_{i}\right)\right)\right. \\
& \left.+\left(1-\Delta_{i}\right) \frac{\int_{Z_{i}}^{+\infty}\left(y-m_{\theta_{0}}\left(X_{i}\right)\right) J\left(F\left(y \mid X_{i}\right)\right) d F\left(y \mid X_{i}\right)}{1-F\left(Z_{i} \mid X_{i}\right)}\right\}+o_{P}\left(n^{-1 / 2}\right) \\
= & R_{21 n k}+R_{22 n k}+o_{P}\left(n^{-1 / 2}\right),
\end{aligned}
$$

$k=1, \ldots, d$. Developing $R_{21 n k}$ leads to

$$
R_{21 n k}=-\frac{2}{n^{2} a_{n}} \sum_{i \neq j} K\left(\frac{X_{i}-X_{j}}{a_{n}}\right) \chi_{k}\left(V_{i}, Z_{j}, \Delta_{j}\right)+o_{P}\left(n^{-1 / 2}\right),
$$

where $V_{i}=\left(X_{i}, Z_{i}, \Delta_{i}\right)$. Next, we rewrite $R_{21 n k}$ as

$$
\begin{aligned}
R_{21 n k}= & \frac{-2}{n^{2} a_{n}} \sum_{i \neq j}\left\{A_{k}^{*}\left(V_{i}, V_{j}\right)+E\left[A_{k}\left(V_{i}, V_{j}\right) \mid V_{i}\right]+E\left[A_{k}\left(V_{i}, V_{j}\right) \mid V_{j}\right]-E\left[A_{k}\left(V_{i}, V_{j}\right)\right]\right\} \\
& \quad+o_{P}\left(n^{-1 / 2}\right) \\
= & T_{1, k}^{n}+T_{2, k}^{n}+T_{3, k}^{n}+T_{4, k}^{n}+o_{P}\left(n^{-1 / 2}\right),
\end{aligned}
$$

where

$$
A_{k}\left(V_{i}, V_{j}\right)=K\left(\frac{X_{i}-X_{j}}{a_{n}}\right) \chi_{k}\left(V_{i}, Z_{j}, \Delta_{j}\right)
$$


and $A_{k}^{*}\left(V_{i}, V_{j}\right)=A_{k}\left(V_{i}, V_{j}\right)-E\left[A_{k}\left(V_{i}, V_{j}\right) \mid V_{i}\right]-E\left[A_{k}\left(V_{i}, V_{j}\right) \mid V_{j}\right]+E\left[A_{k}\left(V_{i}, V_{j}\right)\right]$. Consider

$$
\begin{aligned}
& E\left[A_{k}\left(V_{i}, V_{j}\right) \mid V_{i}\right] \\
& =\sum_{\delta=0,1} \iint \chi_{k}\left(V_{i}, z, \delta\right) K\left(\frac{X_{i}-x}{a_{n}}\right) h_{\delta}(z \mid x) f_{X}(x) d z d x \\
& =a_{n} \sum_{\delta=0,1} \iint \chi_{k}\left(V_{i}, z, \delta\right) K(u)\left(h_{\delta}\left(z \mid X_{i}\right)-a_{n} u \dot{h}_{\delta}\left(z \mid X_{i}\right)+O\left(a_{n}^{2}\right)\right) \\
& \quad \times\left(f_{X}\left(X_{i}\right)-a_{n} u f_{X}^{\prime}\left(X_{i}\right)+O\left(a_{n}^{2}\right)\right) d z d u \\
& =a_{n} f_{X}\left(X_{i}\right) \sum_{\delta=0,1} \int \chi_{k}\left(V_{i}, z, \delta\right) h_{\delta}\left(z \mid X_{i}\right) d z+O\left(a_{n}^{3}\right)=O\left(a_{n}^{3}\right)
\end{aligned}
$$

for $i=1, \ldots, n$, since

$$
\sum_{\delta=0,1} \int \xi(z, \delta, y \mid x) h_{\delta}(z \mid x) d z=0
$$

for all $x \in R_{X}$ and $y \leq T_{x}$. Hence, we also have $E\left[A_{k}\left(V_{i}, V_{j}\right)\right]=O\left(a_{n}^{3}\right)$. In a similar way, using three Taylor expansions of order 2 , we get

$$
\begin{aligned}
E\left[A_{k}\left(V_{i}, V_{j}\right) \mid V_{j}\right]= & a_{n} f_{X}\left(X_{j}\right) \sum_{\delta=0,1} \int \chi_{k}\left(\left(X_{j}, z, \delta\right),\left(Z_{j}, \Delta_{j}\right)\right) d H_{\delta}\left(z \mid X_{j}\right) \\
& +O\left(a_{n}^{3}\right) .
\end{aligned}
$$

Note that for $T_{1, k}^{n}, E\left[T_{1, k}^{n}\right]=0$, resulting, by Chebyshev's inequality, in

$$
\begin{aligned}
P\left(\left|T_{1, k}^{n}\right|>K\left(n a_{n}\right)^{-1}\right) & \leq K^{-2}\left(n a_{n}\right)^{2} E\left[\left(T_{1, k}^{n}\right)^{2}\right] \\
& =4 K^{-2} n^{-2} \sum_{j \neq i} \sum_{m \neq l} E\left[A_{k}^{*}\left(V_{i}, V_{j}\right) A_{k}^{*}\left(V_{l}, V_{m}\right)\right],
\end{aligned}
$$

for any $K>0$. Since $E\left[A_{k}^{*}\left(V_{i}, V_{j}\right)\right]=0$, the terms for which $i, j \neq l, m$ are zero. The terms for which either $i$ or $j$ equals $l$ or $m$ and the other differs from $l$ and $m$, are also zero, because, for example when $i=l$ and $j \neq m$,

$$
E\left[A_{k}^{*}\left(V_{i}, V_{j}\right) E\left[A_{k}^{*}\left(V_{i}, V_{m}\right) \mid V_{i}, V_{j}\right]\right]=0 .
$$

Thus, only the $2 n(n-1)$ terms for which $(i, j)$ equals $(l, m)$ or $(m, l)$ remain. Since $A_{k}^{*}\left(V_{i}, V_{j}\right)$ is bounded by $C K\left(\frac{X_{i}-X_{j}}{a_{n}}\right)+O\left(a_{n}\right)$ for some constant $C>0$, we have (in the case $(i, j)$ equals $(l, m))$ that

$$
E\left[A_{k}^{*^{2}}\left(V_{i}, V_{j}\right)\right] \leq C^{2} a_{n} \int f_{X}^{2}(x) d x \int K^{2}(u) d u+O\left(a_{n}^{2}\right)=O\left(a_{n}\right) .
$$


The case $(i, j)$ equals $(m, l)$ is treated similarly. It now follows that

$$
T_{1, k}^{n}=o_{P}\left(n^{-1} a_{n}^{-1}\right)
$$

which is $o_{P}\left(n^{-1 / 2}\right)$. By (A.2), (A.3), (A.4), we finally obtain

$$
\begin{aligned}
R_{21 n k}= & -\frac{2}{n} \sum_{i=1}^{n} \sum_{\delta=0,1} \int \chi_{k}\left(\left(X_{i}, z, \delta\right),\left(Z_{i}, \Delta_{i}\right)\right) f_{X}\left(X_{i}\right) d H_{\delta}\left(z \mid X_{i}\right) \\
& +o_{P}\left(n^{-1 / 2}\right), \quad k=1, \ldots, d .
\end{aligned}
$$

Finally, we treat the term $R_{1 n}$.

$$
\begin{aligned}
R_{1 n}=- & \frac{2}{n}\left\{\sum _ { i = 1 } ^ { n } \left[\Delta_{i}\left(Z_{i}-m_{\theta_{1 n}}\left(X_{i}\right)\right) J\left(\hat{F}\left(Z_{i} \mid X_{i}\right)\right)\right.\right. \\
& \left.+\left(1-\Delta_{i}\right) \frac{\int_{Z_{i}}^{+\infty}\left(y-m_{\theta_{1} n}\left(X_{i}\right)\right) J\left(\hat{F}\left(y \mid X_{i}\right)\right) d \hat{F}\left(y \mid X_{i}\right)}{1-\hat{F}\left(Z_{i} \mid X_{i}\right)}\right] \frac{\partial^{2} m_{\theta_{1 n}}\left(X_{i}\right)}{\partial \theta \partial \theta^{T}} \\
- & \sum_{i=1}^{n}\left[\Delta_{i} J\left(\hat{F}\left(Z_{i} \mid X_{i}\right)\right)+\left(1-\Delta_{i}\right) \frac{\int_{Z_{i}}^{+\infty} J\left(\hat{F}\left(y \mid X_{i}\right)\right) d \hat{F}\left(y \mid X_{i}\right)}{1-\hat{F}\left(Z_{i} \mid X_{i}\right)}\right] \\
= & R_{11 n}\left(\theta_{1 n}, \hat{F}\right)+R_{12 n}\left(\theta_{1 n}, \hat{F}\right) .
\end{aligned}
$$

Using the uniform consistency of the Beran (1981) estimator together with (A6), it is clear that

$$
R_{11 n}\left(\theta_{1 n}, \hat{F}\right)+R_{12 n}\left(\theta_{1 n}, \hat{F}\right)=R_{11 n}\left(\theta_{0}, F\right)+R_{12 n}\left(\theta_{0}, F\right)+o_{P}(1) .
$$

Since $E\left[R_{11 n}\left(\theta_{0}, F\right)\right]=0$ and

$$
E\left[R_{12 n}\left(\theta_{0}, F\right)\right]=2 E\left[\left(\frac{\partial m_{\theta_{0}}(X)}{\partial \theta}\right)\left(\frac{\partial m_{\theta_{0}}(X)}{\partial \theta^{T}}\right)\right],
$$

we obtain that

$$
R_{1 n}=2 E\left[\left(\frac{\partial m_{\theta_{0}}(X)}{\partial \theta}\right)\left(\frac{\partial m_{\theta_{0}}(X)}{\partial \theta^{T}}\right)\right]+o_{P}(1)
$$

This finishes the proof. 


\section{References}

Akritas, M.G. (1996). On the use of nonparametric regression techniques for fitting parametric regression models. Biometrics, 52, 1342-1362.

Aragon, Y., Daouia, A. and Thomas-Agnan, C. (2005). Nonparametric frontier estimation: a conditional quantile-based approach. Econometric Theory, 21, 358-389.

Beran, R. (1981). Nonparametric regression with randomly censored survival data. Technical Report, Univ. California, Berkeley.

Buchinsky, M. and Hahn, J. (1998). An alternative estimator for the censored quantile regression model. Econometrica, 66, 653-671.

Cazals, C., Florens, J.P. and Simar, L. (2002). Nonparametric frontier estimation: a robust approach. Journal of Econometrics, 106, 1-25.

Fygenson, M. and Zhou, M. (1994). On using stratification in the analysis of linear regression models with right censoring. Annals of Statistics, 22, 747-762.

Heuchenne, C. and Van Keilegom, I. (2007). Nonlinear regression with censored data. Technometrics, 49, 34-44.

Jennrich, R.I. (1969). Asymptotic properties of nonlinear least squares estimators. Annals of Mathematical Statistics, 40, 633-643.

Pardo-Fernández, J.C., Van Keilegom, I. and González-Manteiga, W. (2007). Goodnessof-fit tests for parametric models in censored regression. Canadian Journal of Statistics, 35, 249-264.

Portnoy, S. (2003). Censored regression quantiles. Journal of the American Statistical Association, 98, 1001-1012.

Stute, W. (1993). Consistent estimation under random censorship when covariables are present. Journal of Multivariate Analysis, 45, 89-103.

Van der Vaart, A.W. (1998). Asymptotic statistics. Cambridge University Press, Cambridge.

Van Keilegom, I. and Akritas, M. G. (1999). Transfer of tail information in censored regression models. Annals of Statistics, 27, 1745-1784.

Van Keilegom, I. and Akritas, M. G. (2000). The least squares method in heteroscedastic censored regression models. In : Asymptotics in Statistics and Probability, Ed. M.L. Puri, VSP, 379-391.

Yin, G., Zeng, D. and Li, H. (2008). Power-transformed linear quantile regression with censored data. Journal of the American Statistical Association, 103, 1214-1224. 
Wang, J. and Wang, L. (2009). Locally weighted censored quantile regression. Journal of the American Statistical Association, 104, 1117-1128. 